\title{
Manejo de la hemorragia digestiva alta en pacientes con infección por SARS-CoV-2 en un hospital de Lima, Perú
}

\section{Management of upper gastrointestinal bleeding in patients with SARS-COV-2 infection in a hospital in Lima-Peru}

\author{
Henry Tomas Vargas-Marcacuzco, ${ }^{\text {* }}$ (1) Isamar Benyi Gutiérrez-Córdova, ${ }^{1}$ (1) Sonia Irene Junes-Pérez, ${ }^{1}$ (])

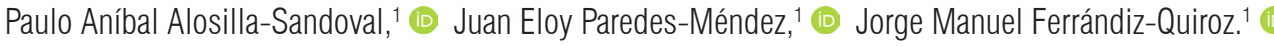

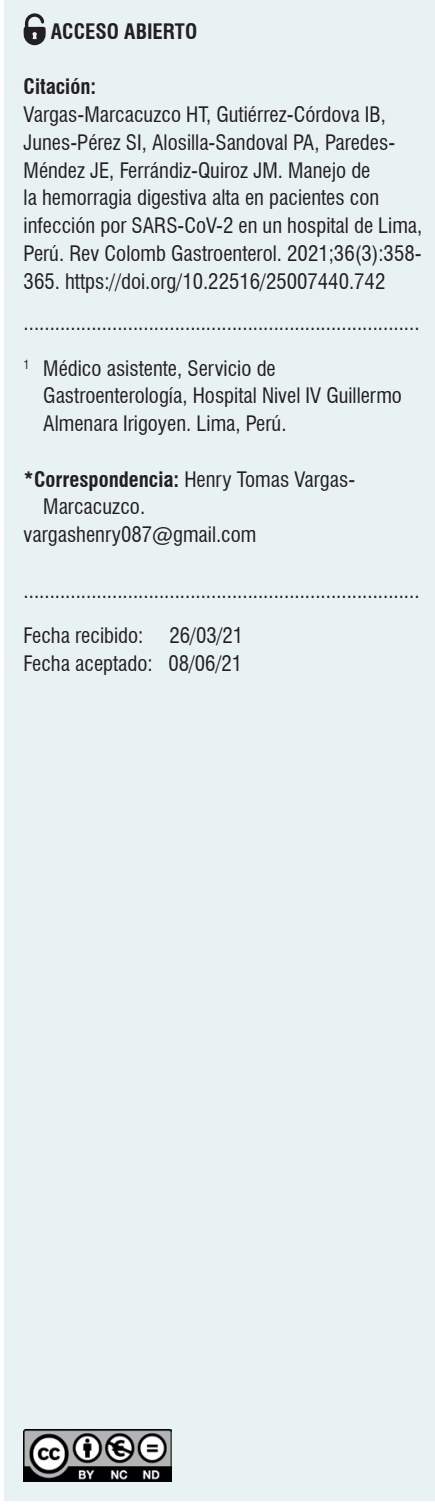

\section{Resumen}

Introducción: la pandemia por SARS-CoV-2 ha conllevado la reestructuración de las unidades de endoscopía digestiva en el mundo, lo cual ha limitado los procedimientos endoscópicos y priorizado indicaciones de emergencia como la hemorragia digestiva alta (HDA). No obstante, existe escasa evidencia respecto a su impacto en la evolución y resultados. Objetivo: evaluar el manejo de la HDA en el contexto de la pandemia del coronavirus por SARS-CoV-2. Materiales y métodos: estudio observacional, descriptivo, retrospectivo de marzo a agosto de 2020 en pacientes con diagnóstico de HDA e infección por SARS-CoV-2. Resultados: de 4320 pacientes con infección por SARS-CoV-2, 51 presentaron HDA al ingreso. La mediana de edad fue de 70 años. El 58,8 \% era de sexo masculino. El 56,9 \% tenía una puntuación de Glasgow-Blatchford (SGB) $\geq 12$. El 21,6 \% requirió soporte de oxígeno. Solo 34 pacientes $(66,7 \%)$ recibieron tratamiento médico; asimismo, $17(33,3 \%)$ recibieron tratamiento médico más endoscopia digestiva alta (EDA); de estos, a $6(35,3 \%)$ se les realizó endoscopia terapéutica. La enfermedad ulcerosa péptica fue el hallazgo más frecuente. Al comparar el tipo de tratamiento recibido, no hubo diferencias significativas entre el número de transfusiones de glóbulos rojos, resangrado, reingreso por HDA, estancia hospitalaria ni mortalidad secundaria a la HDA. La mortalidad global fue del $25,4 \%$ (13 pacientes) y se debió, principalmente, al compromiso respiratorio por SARS-CoV-2. Conclusiones: se observa una reducción en el número de EDA de emergencia por HDA en la pandemia actual, así como un tiempo mayor al estándar para su realización. Más del $80 \%$ de los pacientes que recibieron solo tratamiento médico evolucionaron favorablemente, y solo un tercio de los pacientes a quienes se les realizó una EDA requirió terapéutica endoscópica.

Palabras clave

Hemorragia gastrointestinal, SARS-CoV-2, tratamiento, endoscopia.

\section{Abstract}

Introduction: The SARS-CoV-2 pandemic has led to the restructuring of digestive endoscopy units around the world, limiting endoscopic procedures and prioritizing emergency indications such as upper gastrointestinal hemorrhage (UGH). However, there is little evidence regarding its impact on evolution and outcomes. Objective: To evaluate the management of UGH in the context of the SARS-CoV-2 coronavirus pandemic. Materials and methods: Observational, descriptive, retrospective study carried out between March and August 2020 in patients with diagnosis of UGH and SARS-CoV-2 infection. Results: Of 4320 patients with SARS-CoV-2 infection, 51 had UGH on admission. The median age of the population was 70 years and $58.8 \%$ were male. Glasgow-Blatchford Bleeding Score (GBS) of $\geq 12$ was obtained in $56.9 \%$. Oxygen support was required by $21.6 \%$. $34(66.7 \%)$ patients received medical treatment only, while $17(33.3 \%)$ received medical treatment plus upper gastrointestinal endoscopy (UGE), of which $6(35.3 \%)$ underwent therapeutic endoscopy. Peptic ulcer disease was the most frequent finding. When comparing the type of treatment received, there were no significant differences between the number of red blood cell transfusions, rebleeding, re-admission due to UGH, hospital stay, or mortality secondary to UGH. Overall mortality was $25.4 \%$ (13 patients), mainly due to respiratory failure due to SARS-CoV-2. Conclusions: A reduction in the number of emergency upper gastrointestinal endoscopies for UGH was observed during the current pandemic, as well as a longer than standard time for their performance. More than $80 \%$ of patients who received medical treatment alone evolved favorably and only one third of the patients who underwent UGE required endoscopic therapy.

Keywords

Gastrointestinal bleeding; SARS-CoV-2; Treatment; Endoscopy. 


\section{INTRODUCCIÓN}

La infección producida por el coronavirus SARS-CoV-2 constituye un serio problema de salud actual y de gran impacto global. Su rápida transmisión y evolución desde su origen en diciembre de $2019^{(1,2)}$ hizo que sea declarada como pandemia por la Organización Mundial de la Salud $(\mathrm{OMS})^{(3)}$. En la actualidad se reportan más de 160 millones de casos confirmados y 3,5 millones de pérdidas humanas en todo el mundo ${ }^{(4)}$.

Esta situación ha promovido la reestructuración de los servicios hospitalarios en todo el mundo, incluida la metodología de trabajo en las unidades de endoscopía digestiva (UED). Debido a la generación de aerosoles, la endoscopia expone de manera inadvertida a los trabajadores de salud al contagio mediante la inhalación, transmisión fecal-oral o contacto conjuntival de microgotas o secreciones contaminadas ${ }^{(5,6)}$. En ese sentido, las sociedades mundiales de endoscopia y grupos de expertos recomiendan limitar los procedimientos endoscópicos electivos, siendo prioridad las indicaciones de emergencia ${ }^{(7-10)}$.

La hemorragia digestiva alta (HDA) es una condición potencialmente mortal que requiere un diagnóstico y un manejo oportunos $^{(11)}$. La endoscopia permite identificar y tratar la fuente del sangrado, por lo que se recomienda habitualmente realizarla dentro de las 24 horas posteriores a la presentación del cuadro ${ }^{(12,13)}$. Sin embargo, la pandemia actual ha traído consigo un dilema respecto a la endoscopia en pacientes con HDA e infección por SARS-CoV-2 ${ }^{(14)}$, lo que sugiere que el riesgo de transmitir el virus podría superar el beneficio otorgado por el procedimiento ${ }^{(15)}$.

Bajo ese contexto, la experiencia emergente sugiere optimizar el tratamiento médico, que incluye monitorización hemodinámica, soporte transfusional restrictivo y farmacoterapia $^{(16)}$, lo cual justifica realizar la endoscopia en casos de inestabilidad hemodinámica ${ }^{(7)} \mathrm{o}$ ante el fracaso de la terapia médica en las primeras 24 horas. A la fecha no existen guías específicas con recomendaciones fuertes basadas en evidencia que evalúen el papel del tratamiento médico conservador y/o abordaje endoscópico respecto a la evolución en los pacientes con infección por SARS-CoV-2 y HDA ${ }^{(17)}$.

El objetivo del estudio fue evaluar el manejo de la HDA en el contexto de la pandemia del coronavirus SARS-CoV-2 en un hospital de referencia de Lima, Perú.

\section{MATERIALES Y MÉTODOS}

Se realizó un estudio observacional, descriptivo, retrospectivo entre marzo y agosto de 2020 en el Hospital Nacional Guillermo Almenara Irigoyen, en Lima, Perú.

Se realizó un censo de los pacientes con diagnóstico de HDA en nuestra unidad, quienes cumplían con los criterios de inclusión: edad $\geq 18$ años, pacientes con resultado de prueba rápida y/o molecular positiva para SARS-CoV-2, con registros de las variables indispensables en la historia clínica como: signos de HDA (hematemesis y melena) al ingreso, hemoglobina sérica, registro de transfusión de hemoderivados, tratamiento recibido y evolución. Se excluyeron: mujeres gestantes, pacientes que solicitaron retiro voluntario antes de las 24 horas o que no autorizaron la transfusión de hemoderivados ni procedimientos endoscópicos cuando estaba indicado.

Se recolectaron los datos de las historias clínicas, y la fecha del final del seguimiento fue el día de alta hospitalaria, la derivación a otro centro o la muerte.

Se consideraron como positivos para SARS-CoV-2 aquellos pacientes con prueba serológica reactiva de inmunoglobulina $M(\operatorname{IgM})$ o prueba positiva de reacción en cadena de la polimerasa (PCR) en tiempo real de hisopado nasofaríngeo; sintomáticos o asintomáticos, estos últimos incluso con tomografía de tórax normal ante la posibilidad de encontrarse dentro del período infeccioso de este virus (alta transmisibilidad y patogenicidad) ${ }^{(18,19)}$. Estos pacientes fueron aislados al igual que los sintomáticos.

La puntuación de Glasgow-Blatchford (SGB) fue calculada al ingreso; un valor $\geq 3$ se consideró como indicación de admisión hospitalaria $\mathrm{y} \geq 12$ de alta probabilidad de requerir tratamiento endoscópico y riesgo de muerte ${ }^{(12)}$.

La enfermedad por SARS-CoV-2 fue clasificada de acuerdo con los criterios establecidos por la $\mathrm{OMS}^{(20)}$ :

- leve: paciente sintomático, pero no presenta neumonía ni hipoxemia;

- moderada: neumonía con saturación de oxígeno $\left(\mathrm{O}_{2}\right) \geq$ $90 \%$ al aire ambiental;

- severa: frecuencia respiratoria $\geq 30 / \mathrm{min}$, saturación de $\mathrm{O}_{2}<90 \%$ o presión arterial de oxígeno/fracción inspirada de oxígeno $\left(\mathrm{PaO}_{2} / \mathrm{FiO}_{2}\right)<300 \mathrm{~mm} \mathrm{Hg}$;

- crítica: síndrome de dificultad respiratoria aguda, sepsis o choque séptico.

El manejo médico para la HDA consistió en soporte hemodinámico (administración de solución salina fisiológica con o sin necesidad de hemoderivados) y farmacoterapia (que incluyó un inhibidor de bomba de protones [IBP] como el omeprazol intravenoso de $80 \mathrm{mg}$ en bolo, seguido de una infusión de $8 \mathrm{mg}$ /hora o bolos de $40 \mathrm{mg}$ cada 12 horas). Por otro lado, aquellos con sospecha clínica, de laboratorio o diagnóstico de cirrosis hepática recibieron, como parte del manejo de la HDA varicosa, ocreotida de $50 \mu \mathrm{g}$ intravenosa en bolo, seguida de $50 \mu \mathrm{g} /$ hora y un antibiótico a modo de profilaxis con ceftriaxona de $1 \mathrm{~g} / 24 \mathrm{~h}$ intravenosa.

La indicación de endoscopia digestiva alta (EDA) se consideró ante la sospecha de sangrado activo antes o después 
de las 24 horas de la admisión, y se realizó según las recomendaciones internacionales y criterio médico. Además, la inestabilidad hemodinámica se consideró como una indicación de EDA temprana (entre 6-24 horas) (Figura 1). El tiempo hasta la EDA se determinó según las horas transcurridas desde el ingreso a emergencias por HDA hasta el procedimiento.

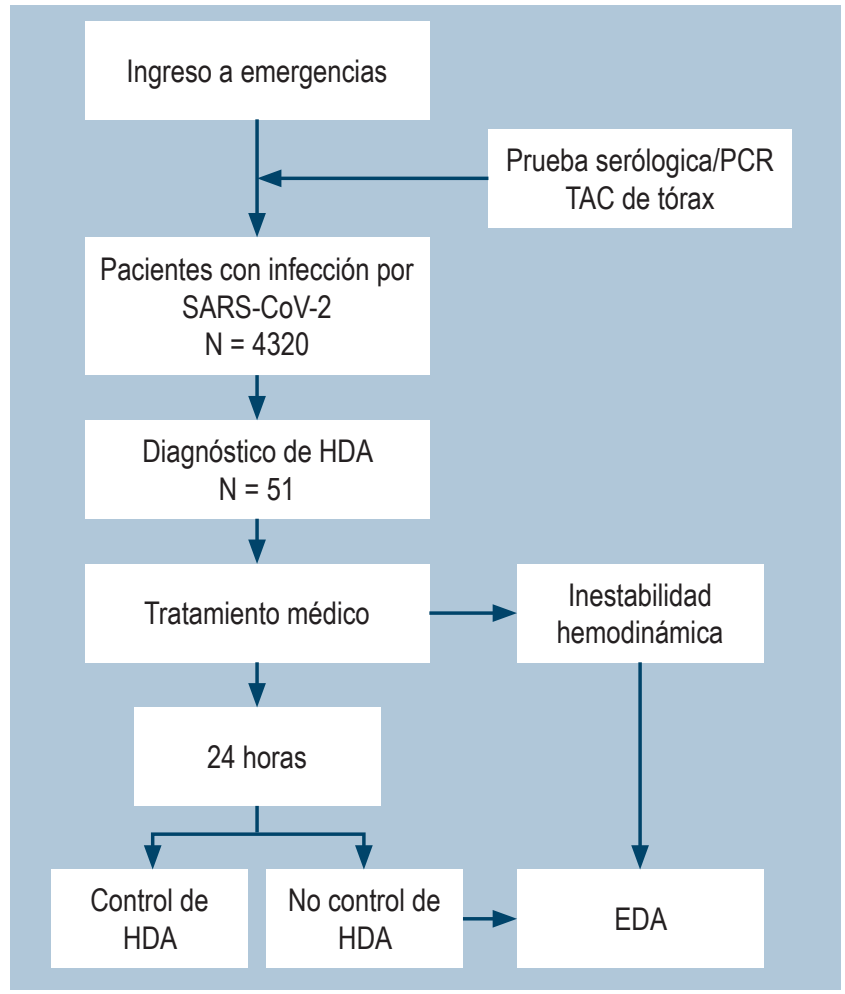

Figura 1. Flujograma de pacientes que ingresaron a emergencias debido a la infección por SARS-CoV-2 y HDA. TAC: tomografía axial computarizada.

Se definió como resangrado cualquier episodio nuevo de HDA dentro de los primeros 5 días luego de evidenciar la ausencia del sangrado digestivo (clínico y bioquímico). Todos los pacientes a quienes no se les realizó una endoscopia en el internamiento inicial fueron dados de alta y se le indicó regresar al centro hospitalario 21 días luego de la prueba inicial positiva para la evaluación ulterior y la programación de una EDA diagnóstica; asimismo, se les indicó el retorno inmediato si presentaban signos de HDA. Si este nuevo episodio reapareció al mes del alta hospitalaria, se consideró como reingreso.

El estudio fue aprobado por el Comité de Ética en Investigación específico para COVID-19 del Instituto de Evaluación de Tecnologías en Salud e Investigación (IETSI) del Seguro Social-EsSalud. Se aplicaron medidas de estadística descriptiva; de las variables categóricas se obtuvieron las frecuencias absolutas y relativas (porcenta- jes). La distribución de los datos fue evaluada mediante la prueba de Kolmogórov-Smirnov. Para las variables cuantitativas se estimaron medidas de tendencia central como la mediana y rango intercuartílico (RIQ). Se compararon variables como: transfusión de hemoderivados, estancia hospitalaria, resangrado, reingreso por HDA y mortalidad específica entre los pacientes que recibieron tratamiento conservador frente al tratamiento endoscópico; para esto se utilizó la prueba U de Mann-Whitney, la prueba chi cuadrado $\left(\chi^{2}\right)$ o la prueba exacta de Fisher, respectivamente. Todos los análisis estadísticos se realizaron con el programa SPSS (Statistical Packages for the Social Sciences) versión 25.0, y las tablas y figuras fueron construidas con Microsoft Excel, 2016. Un valor de $p<0,05$ se consideró como estadísticamente significativo.

\section{RESULTADOS}

De 4320 casos de pacientes con infección por SARS-CoV-2, $51(1,18 \%)$ pacientes presentaron HDA al ingreso a emergencias. La mediana de edad fue de 70 años RIQ (61-77). $30(58,8 \%)$ pacientes eran del sexo masculino. Las comorbilidades más frecuentes fueron hipertensión arterial $(20$ pacientes; $39,2 \%$ ) y cirrosis hepática (12 pacientes; $23,5 \%$ ).

Según la SGB, 51 (100\%) pacientes tuvieron indicación de admisión hospitalaria al ingreso (SGB $\geq 3$ ) y 29 (56,9\%) presentaron una mayor probabilidad de tratamiento endoscópico y muerte $(\mathrm{SGB} \geq 12)$. Con respecto a la infección por SARS-CoV-2, 20 (39,2\%) pacientes presentaron neumonía; de estos, $11(21,6 \%)$ pacientes requirieron soporte de oxígeno y solo $1(2 \%)$ recibió ventilación asistida (Tabla 1).

Respecto al manejo de la HDA, a 34 (66,7\%) pacientes no se les realizó EDA y recibieron solo tratamiento médico, y 17 $(33,3 \%)$ recibieron tratamiento médico más EDA. Como tratamiento médico, $47(92,2 \%)$ pacientes recibieron IBP en bolo, 4 (7,8 \%) recibieron IBP en infusión y $14(27,5 \%)$ recibieron ocreotida intravenosa. De los pacientes a quienes se les realizó EDA, solo $6(35,3 \%)$ requirieron alguna intervención terapéutica. A 16 (94,1 \%) pacientes se le realizó la EDA después de las 24 horas del ingreso, con una mediana de 36 horas RIQ (30-49). El hallazgo más frecuente fue la enfermedad ulcerosa péptica en $7(41,2 \%)$ pacientes, seguida por várices esofagogástricas en $3(17,6 \%)$. Las otras etiologías, así como el tratamiento endoscópico recibido, se resumen en la Tabla 2.

$\mathrm{Al}$ analizar entre aquellos pacientes que recibieron solo tratamiento médico y los que recibieron tratamiento médico más EDA, no observamos diferencias estadísticamente significativas entre las tasas de resangrado ( 3 frente a $0, p=0,542$ ); el reingreso por HDA a los 30 días (3 frente a $0, p=0,542)$; de la misma manera, con el número de 
Tabla 1. Características descriptivas de los pacientes con infección por SARS-CoV-2 y HDA

\begin{tabular}{|c|c|}
\hline Características de los pacientes & $\begin{array}{c}\text { SARS-CoV-2 } \\
n=51\end{array}$ \\
\hline Edad, años, mediana (RIQ) & $70(61-77)$ \\
\hline Sexo, masculino $n(\%)$ & $30(58,8 \%)$ \\
\hline $\begin{array}{l}\text { Comorbilidades, n (\%) } \\
\text { - Hipertensión } \\
\text { - Cirrosis hepática } \\
\text { - Diabetes mellitus } \\
\text { - Enfermedad renal crónica } \\
\text { - Enfermedad cardíaca crónica } \\
\text { - Enfermedad neurológica } \\
\text { - Cáncer }\end{array}$ & $\begin{array}{l}20(39,2 \%) \\
12(23,5 \%) \\
10(19,6 \%) \\
6(11,8 \%) \\
7(13,7 \%) \\
5(9,80 \%) \\
4(7,8 \%)\end{array}$ \\
\hline $\begin{array}{l}\text { Medicación ambulatoria, } \mathrm{n}(\%) \\
\text { - AINE } \\
\text { - Anticoagulantes } \\
\text { - Antiplaquetarios }\end{array}$ & $\begin{array}{c}7(13,7 \%) \\
8(15,7 \%) \\
4(7,8 \%)\end{array}$ \\
\hline $\begin{array}{l}\text { Signos de HDA, n (\%) } \\
\text { - Melena } \\
\text { - Hematemesis }\end{array}$ & $\begin{array}{c}37(72,5 \%) \\
26(51 \%)\end{array}$ \\
\hline $\begin{array}{l}\text { SGB, } n(\%) \\
-\geq 3 \\
-\geq 12\end{array}$ & $\begin{array}{l}51(100 \%) \\
29(56,9 \%)\end{array}$ \\
\hline $\begin{array}{l}\text { Hipotensión/choque, n (\%) } \\
\text { - Sí }\end{array}$ & $8(15,6 \%)$ \\
\hline $\begin{array}{l}\text { Saturación de } \mathrm{O}_{2}, \mathrm{n}(\%) \\
-\quad<92 \%\end{array}$ & $11(21,6 \%)$ \\
\hline $\begin{array}{l}\text { Gravedad del SARS-CoV-2, n (\%) } \\
\text { - Infección asintomática } \\
\text { - Enfermedad leve } \\
\text { - Enfermedad moderada } \\
\text { - Enfermedad severa } \\
\text { - Enfermedad crítica }\end{array}$ & $\begin{array}{c}19(37,3 \%) \\
12(23,5 \%) \\
10(19,6 \%) \\
9(17,6 \%) \\
1(2,0 \%)\end{array}$ \\
\hline $\begin{array}{l}\text { Analítica al ingreso, mediana (RIQ) } \\
\text { - Hemoglobina } \\
\text { - Plaquetas } \\
\text { - INR } \\
\text { - Leucocitos } \\
\text { - Linfocitos } \\
\text { - Neutrófilos } \\
\text { - PCR } \\
\text { - Urea } \\
\text { - Creatinina } \\
\text { - Fibrinógeno } \\
\text { - Tiempo de protrombina } \\
\text { - TTPa } \\
\text { - Fosfatasa alcalina } \\
\text { - AST (aspartato-aminotransferasa) } \\
\text { - ALT (alanina-aminotransferasa) } \\
\text { - Gamma-glutamil transpeptidasa } \\
\text { - Bilirrubina total } \\
\text { - Albúmina } \\
\text { - Proteínas totales }\end{array}$ & $\begin{array}{c}8,1(6-11,6) \\
229(149-329) \\
1(0,93-1,32) \\
9790(6090-12430) \\
1249(767-1958) \\
7785(4506-10344) \\
23,1(8,2-55,2) \\
55,2(32,7-102,5) \\
0,9(0,6-1,6) \\
3,8(2,97-4,37) \\
11,06(10,13-14,05) \\
31,86(27,5-38,89) \\
111,5(70,5-146) \\
34(26-50) \\
28(19-47) \\
43,5(21-106,5) \\
0,5(0,3-0,96) \\
3,1(2,8-3,7) \\
5,9(5,3-6,35)\end{array}$ \\
\hline
\end{tabular}

AINE: antiinflamatorios no esteroideos; INR: International Normalized Ratio; PCR: proteína C-reactiva; TTPa: tiempo de tromboplastina parcial activado.
Tabla 2. Manejo médico y/o endoscópico de los pacientes con infección por SARS-CoV-2 y HDA

\begin{tabular}{|c|c|}
\hline Características del manejo & $\begin{array}{c}\text { SARS-CoV2 } \\
n=51\end{array}$ \\
\hline $\begin{array}{l}\text { Solo tratamiento médico, } \mathrm{n}(\%) \\
\text { - IBP en bolo intravenoso } \\
\text { - IBP en infusión intravenoso } \\
\text { - Octreotida en infusión intravenosa } \\
\text { - Ceftriaxona intravenosa } \\
\text { - } \text { Transfusión de glóbulos rojos, } \mathrm{n}(\%) \\
\text { - } \quad \text { Transfusión de plasma fresco, } \mathrm{n}(\%) \\
\text { - } \quad \text { Transfusión de plaquetas, } \mathrm{n}(\%)\end{array}$ & $\begin{array}{c}34(66,7 \%) \\
47(92,2 \%) \\
4(7,8 \%) \\
14(27,5 \%) \\
16(31,4 \%) \\
29(56,9 \%) \\
7(13,7 \%) \\
3(5,9 \%)\end{array}$ \\
\hline Tratamiento médico + endoscopia, $n(\%)$ & $17(33,3 \%)$ \\
\hline Tiempo hasta endoscopia $>24$ horas & $16(94,1 \%)$ \\
\hline $\begin{array}{l}\text { Hallazgos endoscópicos, } \mathrm{n}(\%) \\
\text { - Úlcera duodenal o gástrica } \\
\text { - Várices esofagogástricas } \\
\text { - Gastritis erosiva o hemorrágica } \\
\text { - Neoplasia } \\
\text { - Angiodisplasia } \\
\text { - Mallory-Weiss } \\
\text { - Esofagitis erosiva }\end{array}$ & $\begin{array}{c}7(41,2 \%) \\
3(17,6 \%) \\
2(11,8 \%) \\
1(5,9 \%) \\
2(11,8 \%) \\
1(5,9 \%) \\
1(5,9 \%)\end{array}$ \\
\hline $\begin{array}{l}\text { Tratamiento endoscópico, n (\%) } \\
\text { - Inyección con adrenalina + hemoclips } \\
\text { - Inyección con adrenalina + APC } \\
\text { - Ligadura de várices esofágicas } \\
\text { - Ninguno }\end{array}$ & $\begin{array}{c}2(11,8 \%) \\
1(5,9 \%) \\
3(17,6 \%) \\
11(64,7 \%)\end{array}$ \\
\hline
\end{tabular}

APC: coagulación con argón plasma.

transfusiones de glóbulos rojos [2(0-2) y $2(0-2), p$ $<0,589]$. La estancia hospitalaria fue de 7,5 RIQ (3-15) y 5 RIQ (2-14) días, respectivamente, sin alcanzar una diferencia significativa $(p<0,352)$. La mortalidad secundaria a HDA fue de 2 frente a $0, p=0,546$. La mortalidad por una causa diferente a la HDA se observó en 11 pacientes, 2 por descompensación de enfermedades crónicas subyacentes y 9 por insuficiencia respiratoria aguda secundaria a neumonía por SARS-CoV-2. La mortalidad global a los 30 días fue del 25,4 \% (13 pacientes) (Tabla 3 ).

\section{DISCUSIÓN}

Perú es uno de los países más afectados por la pandemia actual debido al gran número de contagios simultáneos y un importante número de personal de salud infectado. Sin embargo, la hemorragia gastrointestinal continúa siendo un motivo de ingreso frecuente en nuestro hospital.

Cada vez existe más evidencia de que la EDA muy temprana no ofrece un gran beneficio en cuanto a la reducción 
Tabla 3. Resultados según el tipo de tratamiento recibido de los pacientes con infección por SARS-CoV-2 y HDA

\begin{tabular}{lccc}
\hline \multicolumn{1}{|c}{ Variables } & $\begin{array}{c}\text { Tratamiento } \\
\text { médico } \\
\text { solamente } \\
\mathbf{n}=34\end{array}$ & $\begin{array}{c}\text { Tratamiento } \\
\text { médico } \\
\text { más } \\
\text { endoscopia } \\
\mathbf{n = 1 7}\end{array}$ & $p$ \\
\hline Resangrado, $\mathbf{n}(\%)$ & $3(8,82 \%)$ & $0(0,0 \%)$ & $0,542^{+}$ \\
\hline Reingreso por HDA, $\mathbf{n}(\%)$ & $3(8,82 \%)$ & $0(0,0 \%)$ & $0,542^{+}$ \\
\hline $\begin{array}{l}\text { Estancia hospitalaria, } \\
\text { mediana (RIQ) }\end{array}$ & $7,5(3-15)$ & $5(2-14)$ & $0,352^{*}$ \\
$\begin{array}{l}\text { Número de transfusiones } \\
\text { de glóbulos rojos, mediana } \\
\text { (RIQ) }\end{array}$ & $2(0-2)$ & $2(0-2)$ & $0,589^{*}$ \\
$\begin{array}{l}\text { Mortalidad secundaria a } \\
\text { HDA, } \mathbf{n}(\%)\end{array}$ & $2(5,88 \%)$ & $0(0,0 \%)$ & $0,546+$ \\
\hline
\end{tabular}

*U de Mann-Whitney; ${ }^{+}$test exacto de Fisher

de la mortalidad. Un estudio realizado por Laursen y colaboradores, con una cohorte de 12601 pacientes con HDA, no encontró una asociación significativa entre el tiempo de endoscopia y la mortalidad hospitalaria en pacientes estables hemodinámicamente y sin comorbilidades. Solo se observó un beneficio en la endoscopia temprana (entre las 6 y 24 horas) en aquellos con inestabilidad hemodinámica $^{(21)}$. Lau y colaboradores, en un estudio de 516 pacientes con HDA, describieron que no hubo una diferencia significativa en cuanto a la mortalidad a los 30 días entre pacientes que presentaban un SGB $>12$ y a quienes se le realizó una endoscopia urgente (antes de las 6 horas) frente a temprana (entre 6 y 24 horas) ${ }^{(12)}$.

En la actualidad existe un número reducido de reportes que evalúen el impacto de la pandemia por SARS-CoV-2 en el manejo de la HDA.

En nuestro estudio, la mediana de edad fue de 70 años y el $58,8 \%$ eran de sexo masculino, datos semejantes a los reportados por Martin y colaboradores en un estudio de 31 pacientes con HDA e infección por SARS-CoV-2 en el cual la edad media fue de 68,7 años y el $66 \%$ eran varones ${ }^{(22)}$. Shalimar y colaboradores reportaron que de 1342 pacientes con infección por SARS-CoV-2, 24 pacientes $(1,8 \%)$ presentaron HDA y $70,8 \%$ eran varones ${ }^{(14)}$; no obstante, la edad media fue de 45 años, significativamente menor a la de nuestro estudio.

En nuestra serie, las comorbilidades más frecuentes fueron hipertensión arterial y cirrosis hepática, lo que coincide con otros reportes. En un estudio en Italia, Mauro y colaboradores, de 4871 pacientes con infección por SARS-CoV-2,
$23(0,47 \%)$ tuvieron HDA; de estos, la comorbilidad más frecuente fue hipertensión arterial $(70 \%)^{(23)}$. Asimismo, Martin y colaboradores también encontraron la hipertensión arterial (66\%) comola comorbilidad más prevalente ${ }^{(22)}$. Por otro lado, Shalimar y colaboradores reportaron que casi la totalidad de sus pacientes eran cirróticos $(91 \%)^{(14)}$. El gran porcentaje de pacientes en nuestro estudio con hepatopatía crónica podría deberse a que nuestro hospital es un centro de referencia nacional de pacientes con comorbilidades complejas.

Debemos resaltar que el $56,9 \%$ de nuestros pacientes presentaban un riesgo elevado de necesitar terapia endoscópica y de muerte al presentar un $S G B \geq 12$. Asimismo, el 39,2 \% presentaron neumonía y el 21,6 \% requirieron soporte con oxígeno suplementario. Mauro y colaboradores reportaron que de 23 pacientes con $\mathrm{HDA}$, el $82 \%$ requirió soporte con oxígeno suplementario, con una mediana de SGB de 13 puntos RIQ (10-16) ${ }^{(23)}$. Martin y colaboradores informaron, en 31 pacientes con HDA, que el $39 \%$ necesitó intubación o admisión a la unidad de cuidados intensivos (UCI), con una media SGB de 10 puntos $^{(22)}$.

Se han publicado algunos reportes de casos sobre el manejo de los pacientes con HDA e infección por SARS$\mathrm{CoV}-2$. Cavaliere y colaboradores reportaron 6 pacientes, 5 de ellos con necesidad de soporte de oxígeno y 1 con ventilación asistida. Todos respondieron al manejo médico conservador y no se les realizó una $\mathrm{EDA}^{(15)}$. Gadiparthi y colaboradores reportaron 3 casos, 2 de ellos fueron HDA con requerimientos de oxígeno suplementario y $S G B \geq 7$, en los cuales se resolvió el cuadro solamente con tratamiento médico conservador; recomiendan considerar este manejo si es poco probable que la intervención endoscópica mejore el resultado (pacientes muy estables o críticos) ${ }^{(24)}$. Barrett y colaboradores reportaron 4 casos de pacientes con HDA, todos fueron manejados con tratamiento médico conservador y a 1 se le realizó una endoscopia sin necesidad de una intervención terapéutica ${ }^{(25)}$. Todos coinciden en que el tratamiento médico conservador es una opción válida de manejo ${ }^{(15,24,25)}$ bajo la premisa de que el riesgo de la endoscópica podría superar el beneficio, sobre todo en el contexto actual, donde salvaguardar al personal de salud es fundamental ${ }^{(24)}$.

Kim y colaboradores, en un estudio de cohorte retrospectiva realizado en Nueva York, analizaron 211 pacientes que ingresaron por HDA ( $42 \%$ de ellos durante la pandemia) y reportaron que solo $28 \%$ fueron sometidos al procedimiento $^{(26)}$. En nuestra serie, a 33,3\% de nuestros pacientes se les indicó EDA, con un intervalo de tiempo hasta la realización del procedimiento $>24$ horas en el 94,1\%. Estos hallazgos son similares a los de Mauro y colaboradores, quienes realizaron EDA en el 78,2 \% de sus pacientes con un tiempo promedio $>24$ horas $(2-60 \text { horas })^{(23)}$. Por 
su parte, Martin y colaboradores informaron una demora promedio de 2,4 \pm 2 días en el $32 \%$ de pacientes a quienes se les realizó el procedimiento ${ }^{(22)}$. Estos resultados variables probablemente se deben a que, al tratarse de una enfermedad nueva con gran potencial de contagio para el personal de salud, la indicación se limitaría a los casos seleccionados netamente necesarios, y la demora estaría relacionada con la preparación de toda la logística necesaria recomendada para la realización del procedimiento ${ }^{(27)}$.

La enfermedad ulcerosa péptica y las várices esofagogástricas fueron los hallazgos endoscópicos más frecuentes, similar a lo encontrado en estudios publicados recientemente de HDA e infección por SARS-CoV-2. Por otro lado, se requirió intervención terapéutica endoscópica solamente en un tercio de nuestros pacientes, resultados similares a los reportados por estos autores $(38 \%-40 \%)^{(22,23)}$. Este bajo porcentaje terapéutico se debe probablemente al intervalo de tiempo transcurrido desde el evento hasta el procedimiento, en donde el tratamiento médico también desempeña un papel importante en el manejo.

Durante la evolución, 3 de nuestros pacientes tratados previamente con manejo médico presentaron resangrado. A todos se les realizó endoscopia; 2 pacientes tenían enfermedad ulcerosa péptica (recibieron doble terapia endoscópica) y 1 tenía cáncer gástrico avanzado. Mauro y colaboradores también observaron resangrado en 3 pacientes, de los cuales 2 se consideraron aptos para una embolización radiológica y 1 recibió terapia endoscópica ${ }^{(23)}$. Por otra parte, Martin y colaboradores identificaron resangrado en 4 pacientes durante la misma hospitalización, aunque ninguno requirió intervención endoscópica; mientras que Shalimar y colaboradores ${ }^{(22)}$ informaron dos resangrados, uno recibió terapia endoscópica con argón plasma por presentar ectasia vascular gástrica antral (GAVE) y el otro tuvo una resolución espontánea del sangrado y no requirió $\mathrm{EDA}^{(14)}$. Por otro lado, a aquellos pacientes de nuestra serie que reingresaron por HDA y habían recibido solo tratamiento médico se les realizó una EDA en la nueva admisión hospitalaria, en la que se encontraron várices esofágicas con estigmas de sangrado reciente en 2 pacientes y enfermedad ulcerosa péptica en 1; a todos se les realizó una endoscopia terapéutica con buena evolución.

Kim y colaboradores observaron 2,86 veces más probabilidad de recibir una transfusión de paquetes globulares en los pacientes con ingreso hospitalario por HDA durante la pandemia $(p=0,013)$, así como 2,5 veces más probabilidad de estancia hospitalaria $\geq 5$ días $(p=0,004)$ en comparación con el período prepandemia ${ }^{(26)}$. A su vez, Shalimar y colaboradores señalaron una media de estancia hospitalaria de $7,5(5-12,7)$ días, similar a nuestro hallazgo ${ }^{(14)}$. En nuestro estudio, al comparar entre aquellos pacientes que recibieron tratamiento médico conservador y tratamiento médico más EDA observamos que, a pesar de existir una diferencia numérica entre las variables estudiadas (resangrado, reingreso por HDA, estancia hospitalaria, número de transfusiones de glóbulos rojos, mortalidad debida a HDA), estas no alcanzaron significancia estadística. En relación, Martin y colaboradores no encontraron diferencias sobre la necesidad de transfusión de paquetes globulares $(3,75$ $\pm 3,40$ frente a $3,33 \pm 2,19 ; p=0,777$ ) entre el grupo de tratamiento médico más EDA frente al tratamiento médico conservador ${ }^{(22)}$; del mismo modo, otro estudio señaló una mortalidad similar ( 3 vs. a 2 pacientes) y resangrado ( 2 vs. a 1 paciente), entre ambos grupos ${ }^{(23)}$.

La mortalidad secundaria a HDA en nuestra serie se presentó en 2 pacientes y se debió a choque hipovolémico por hemorragia masiva desde el ingreso. A pesar de intentar la estabilización hemodinámica de los pacientes para la realización del procedimiento, estos fallecieron dentro de las 6 horas siguientes a la admisión. Estudios similares no reportaron mortalidad secundaria a $\operatorname{HDA}^{(22-26)}$, probablemente debido a tamaños muestrales con menor número de casos, lo cual desestimaría la aparición de desenlaces fatales.

La mortalidad global a los 30 días fue similar a otros reportes $(13 \% \text { a } 21 \%)^{(14,23)}$ y se debió principalmente al compromiso respiratorio por SARS-CoV-2.

Dentro de las limitaciones de nuestro estudio es preciso señalar que se realizó en un único centro, con número reducido de casos, además de ser un estudio retrospectivo. Futuros estudios deberían contemplar estos aspectos.

\section{CONCLUSIÓN}

Se observa una reducción en el número de EDA de emergencia por HDA en la pandemia actual, así como un tiempo mayor al estándar para su realización. La enfermedad ulcerosa péptica continúa como la etiología principal de HDA en pacientes con infección por SARS-CoV-2. Más del $80 \%$ de los pacientes que recibieron solo tratamiento médico evolucionaron favorablemente, y solo un tercio de los pacientes a quienes se les realizó una EDA requirió una intervención terapéutica endoscópica.

\section{Conflicto de intereses}

Los autores declaran no tener conflicto de intereses de ningún tipo.

\section{Financiamiento}

La presente investigación no ha recibido ayudas específicas provenientes de agencias del sector público, sector comercial o entidades sin ánimo de lucro. 


\section{REFERENCIAS}

1. WHO | Pneumonia of unknown cause - China. WHO [Internet]. 2020 [consultado el 13 de octubre de 2020]. Disponible en: http://www.who.int/csr/don/05-january2020-pneumonia-of-unkown-cause-china/en/

2. Tan W, Zhao X, Ma X, Wang W, Niu P, Xu W, Cao GF, Wu G. A Novel Coronavirus Genome Identified in a Cluster of Pneumonia Cases - Wuhan, China 2019-2020. China CDC Wkly. 2020;2(4):61-2. https://doi.org/10.46234/ccdcw2020.017

3. WHO. WHO Director-General's opening remarks at themedia briefing on COVID-19 - 11 March 2020 [Internet]. WHO Director General's speeches. 2020. p. 4. [Consultado el 13 de octubre de 2020]. Disponible en: https://www.who.int/dg/speeches/detail/who-directorgeneral-s-opening-remarksat-the-media-briefing-on-covid19---11-march-2020

4. WHO Coronavirus Disease (COVID-19) Dashboard [Internet]. [Consultado el 21 de mayo de 2021]. Disponible en: https://covid19.who.int/

5. Danese S, Ran ZH, Repici A, Tong J, Omodei P, Aghemo A, Malesci A. Gastroenterology department operational reorganisation at the time of covid-19 outbreak: an Italian and Chinese experience. Gut. 2020;69(6):981-83. http://dx.doi.org/10.1136/gutjnl-2020-321143

6. Chan SM, Ma TW, Ka-Chun Chong M, Chan DL, Ng EKW, Chiu PWY. A proof of concept study: Esophagogastroduodenoscopy is an aerosol-generating procedure and continuous oral suction during the procedure reduces the amount of aerosol generated. Gastroenterology. 2020. https://doi.org/10.1053/j.gastro.2020.07.002

7. Gralnek IM, Hassan C, Beilenhoff U, Antonelli G, Ebigbo A, Pellisè M, Arvanitakis M, Bhandari P, Bisschops R, Van Hooft JE, Kaminski MF, Triantafyllou K, Webster G, Pohl H, Dunkley I, Fehrke B, Gazic M, Gjergek T, Maasen S, Waagenes W, de Pater M, Ponchon T, Siersema PD, Messmann H, Dinis-Ribeiro M. ESGE and ESGENA Position Statement on gastrointestinal endoscopy and the COVID-19 pandemic. Endoscopy. 2020;52(6):483-90. https://doi.org/10.1055/a-1155-6229

8. Soetikno R, Teoh AYB, Kaltenbach T, Lau JYW, Asokkumar R, Cabral-Prodigalidad P, Shergill A. Considerations in performing endoscopy during the COVID-19 pandemic. Gastrointest Endosc. 2020;92(1):176-83. https://doi.org/10.1016/j.gie.2020.03.3758

9. Chiu PWY, Ng SC, Inoue H, Reddy DN, Ling Hu E, Cho JY, Ho LK, Hewett DG, Chiu HM, Rerknimitr R, Wang HP, Ho SH, Seo DW, Goh KL, Tajiri H, Kitano S, Chan FKL. Practice of endoscopy during COVID-19 pandemic: position statements of the Asian Pacific Society for Digestive Endoscopy (APSDE-COVID statements). Gut. 2020;69(6):991-6.

http://dx.doi.org/10.1136/gutjnl-2020-321185
10. Castro-Filho EC, Castro R, Fernandes FF, Pereira G, Perazzo H. Gastrointestinal endoscopy during the COVID-19 pandemic: an updated review of guidelines and statements from international and national societies. Gastrointest Endosc. 2020;92(2):440-445.e6. https://doi.org/10.1016/j.gie.2020.03.3854

11. Lanas A, Dumonceau JM, Hunt RH, Fujishiro M, Scheiman JM, Gralnek IM, Campbell HE, Rostom A, Villanueva C, Sung JJY. Non-variceal upper gastrointestinal bleeding. Nat Rev Dis Primers. 2018;4:18020. https://doi.org/10.1038/nrdp.2018.20

12. Lau JYW, Yu Y, Tang RSY, Chan HCH, Yip HC, Chan SM, Luk SWY, Wong SH, Lau LHS, Lui RN, Chan TT, Mak JWY, Chan FKL, Sung JJY. Timing of endoscopy for acute upper gastrointestinal bleeding. N Engl J Med. 2020;382(14):1299-1308. https://doi.org/10.1056/NEJMoa1912484

13. Barkun AN, Almadi M, Kuipers EJ, Laine L, Sung J, Tse F, Leontiadis GI, Abraham NS, Calvet X, Chan FKL, Douketis J, Enns R, Gralnek IM, Jairath V, Jensen D, Lau J, Lip GYH, Loffroy R, Maluf-Filho F, Meltzer AC, Reddy N, Saltzman JR, Marshall JK, Bardou M. Management of Nonvariceal Upper Gastrointestinal Bleeding: Guideline Recommendations From the International Consensus Group. Ann Intern Med. 2019;171(11):805-22. https://doi.org/10.7326/M19-1795

14. Shalimar, Vaishnav M, Elhence A, Kumar R, Mohta S, Palle C, Kumar P, Ranjan M, Vajpai T, Prasad S, Yegurla J, Dhooria A, Banyal V, Agarwal S, Bansal R, Bhattacharjee S, Aggarwal R, Soni KD, Rudravaram S, Singh AK, Altaf I, Choudekar A, Mahapatra SJ, Gunjan D, Kedia S, Makharia G, Trikha A, Garg P, Saraya A. Outcome of Conservative Therapy in Coronavirus disease-2019 Patients Presenting With Gastrointestinal Bleeding. J Clin Exp Hepatol. 2021;11(3):327-333. https://doi.org/10.1016/j.jceh.2020.09.007

15. Cavaliere K, Levine C, Wander P, Sejpal DV, Trindade AJ. Management of upper GI bleeding in patients with COVID-19 pneumonia. Gastrointest Endosc. 2020;92(2):454-55. https://doi.org/10.1016/j.gie.2020.04.028

16. Baradarian R, Ramdhaney S, Chapalamadugu R, Skoczylas L, Wang K, Rivilis S, Remus K, Mayer I, Iswara K, Tenner $\mathrm{S}$. Early intensive resuscitation of patients with upper gastrointestinal bleeding decreases mortality. Am J Gastroenterol. 2004;99(4):619-22. https://doi.org/10.1111/j.1572-0241.2004.04073.x.

17. Aguila EJT, Cua IHY, Raymundo NTV. The Dilemma in the Management of Gastrointestinal Bleeding During the COVID-19 Pandemic. Gastroenterology. 2021;160(5):1889-90. https://doi.org/10.1053/j.gastro.2020.05.088

18. Tong ZD, Tang A, Li KF, Li P, Wang HL, Yi JP, Zhang YL, Yan JB. Potential Presymptomatic Transmission of SARSCoV-2, Zhejiang Province, China, 2020. Emerg Infect Dis. 
2020;26(5):1052-54.

https://doi.org/10.3201/eid2605.200198

19. Deeks JJ, Dinnes J, Takwoingi Y, Davenport C, Spijker

R, Taylor-Phillips S, Adriano A, Beese S, Dretzke J, Ferrante di Ruffano L, Harris IM, Price MJ, Dittrich S, Emperador D, Hooft L, Leeflang MM, Van den Bruel A; Cochrane COVID-19 Diagnostic Test Accuracy Group. Antibody tests for identification of current and past infection with SARS-CoV-2. Cochrane Database Syst Rev. 2020;6(6):CD013652.

https://doi.org/10.1002/14651858.CD013652

20. Clinical management of COVID-19. Geneva: World Health Organization; 2020. [Consultado el 14 de octubre de 2020]. Disponible en: https://www.who.int/ publications/i/item/clinical-management-of-covid-19

21. Laursen SB, Leontiadis GI, Stanley AJ, Møller MH, Hansen JM, Schaffalitzky de Muckadell OB. Relationship between timing of endoscopy and mortality in patients with peptic ulcer bleeding: a nationwide cohort study. Gastrointest Endosc. 2017;85(5):936-944.e3. https://doi.org/10.1016/j.gie.2016.08.049

22. Martin TA, Wan DW, Hajifathalian $K$, Tewani S, Shah SL, Mehta A, Kaplan A, Ghosh G, Choi AJ, Krisko TI, Fortune BE, Crawford CV, Sharaiha RZ. Gastrointestinal Bleeding in Patients With Coronavirus Disease 2019: A Matched Case-Control Study. Am J Gastroenterol. 2020;115(10):1609-16. https://doi.org/10.14309/ajg.0000000000000805
23. Mauro A, De Grazia F, Lenti MV, Penagini R, Frego R, Ardizzone S, Savarino E, Radaelli F, Bosani M, Orlando S, Amato A, Dinelli M, Ferretti F, Filippi E, Vecchi M, Stradella D, Bardone M, Pozzi L, Rovedatti L, Strada E, Di Sabatino A. Upper gastrointestinal bleeding in COVID-19 inpatients: Incidence and management in a multicenter experience from Northern Italy. Clin Res Hepatol Gastroenterol. 2021;45(3):101521. https://doi.org/10.1016/j.clinre.2020.07.025

24. Gadiparthi C, Perisetti A, Sayana H, Tharian B, Inamdar S, Korman A. Gastrointestinal Bleeding in Patients with Severe SARS-CoV-2. Am J Gastroenterol. 2020;115(8):1283-85. https://doi.org/10.14309/ajg.0000000000000719

25. Barrett LF, Lo KB, Stanek SR, Walter JW. Self-limited gastrointestinal bleeding in COVID-19. Clin Res Hepatol Gastroenterol. 2020;44(4):e77-e80. https://doi.org/10.1016/j.clinre.2020.06.015

26. Kim J, Doyle JB, Blackett JW, May B, Hur C, Lebwohl $B$; HIRE study group. Effect of the Coronavirus 2019 Pandemic on Outcomes for Patients Admitted With Gastrointestinal Bleeding in New York City. Gastroenterology. 2020;159(3):1155-57.e1. https://doi.org/10.1053/j.gastro.2020.05.031

27. Perisetti A, Goyal H, Sharma N. Gastrointestinal Endoscopy in the Era of COVID-19. Front Med 2020;7:587602. https://doi.org/10.3389/fmed.2020.587602 